\title{
Work-Health Balance: Characterizing Short-Term and Long-Term Impact to Health
}

\author{
Tabya Sultan \\ Katal Think Labs \\ California \\ USA
}

\author{
Nitish Nag Ph.D. \\ Katal Think Labs \\ California \\ USA
}

\begin{abstract}
People who are motivated to live a healthy lifestyle face competing priorities and resources that interfere with healthy living. A key component of this challenge is maintaining what we call, work-health balance, the optimal state for one's work stress level and biological outcomes. The lack of balance, surpassing one's ideal eustress level, ultimately impacts a user's total health state given changes to the user's lifestyle, or everyday actions, such as eating, sleeping, moving, stress, and other mental preoccupations. We first explore specifically the conflicting dynamic of a user's psychological demands of work and the impact on the user's biological health state. The health state is impacted both in the short-term, including changes to lifestyle events and stress levels and also in the longer-term, including in the development of chronic disease. We established a method for characterizing users' work impact on the health state and their longer-term health potential. We conducted an $\mathrm{N}$-of-1 experiment tracking user lifestyle and stress over 51 weeks to characterize how work deadlines impact lifestyle and stress. We find a direct relationship between user work deadlines and their resulting health state. Identifying and quantifying the health state as it relates to work demands gives users insight into work and lifestyle decision-making. Also, it provides employers with considerations for incorporating work-health balance for the well-being of their employees.
\end{abstract}

Index Terms-work-health balance, work life balance, stress, health state estimation, health potential, lifestyle, lifestyle design, healthy lifestyle, personal health navigation

\section{INTRODUCTION}

A commonly overlooked component of healthy living is the nature of competing priorities in one's life. Individuals may have multiple competing priorities, given their resources that ultimately leaves them in an unsustainable lifestyle. This common challenge is widely referred to as work-life balance. Work-life balance is each individual's optimal state for the time allocated towards work and other life domains (i.e., relationships, health, hobbies) to live in a balanced and predictable way. Work-life balance is associated with benefits, including job and life satisfaction [12]. The development of work-life balance strategies assist in providing a healthier lifestyle for employees [8], and is more widely considered in organizational policies for talent retention purposes [1]. However, the lack of balance in work and life domains is a growing problem in the workforce. The Harvard Business School survey found that 94 percent of service professionals put in more than 50 hours a week [21]. The imbalance harms how much workers have to give up their personal time, causing a negative impact on personal relationships [3]. The standard premise is that work-life balance provisions are introduced to help employees reconcile what they want to do, or care about, with what they have to do, or work [25].

Furthermore, while work may be a high priority in one's life, it also contributes to high-stress levels and poor health outcomes as a result of disturbed balance [14]. Individuals face dilemmas to prioritize responsibilities at work or partake in other areas of life, such as healthy habits, hobbies, and relationships. With time as the key limited resource, people have to plan and structure their days around how they spend time on health versus other areas of life. Health becomes a currency that is sacrificed for the sake of other urgent tasks. When the work domain holds prioritization in a way that is not desired by the individual, poor health is usually a result. This ongoing struggle to find a balance brings about both acute and chronic health effects. Various studies have found that perceived job stress, such as job threats, are linked to poor well-being and increased illness [2]. The Finnish Institute of Occupational Health found that overwork and the resulting stress can lead to impaired sleep, depression, heavy drinking, diabetes, impaired memory, and heart disease [4]. For this reason, it is paramount that an individual can understand how their work causes an impact on their personal health state [19], [16].

While there is continuing evidence on work imbalance and health impact, there remains a perceptual struggle with the demands of one's career and personal life. Threats or urgent needs seem to be high priorities, and individuals face "eitheror" situations of completing an urgent task immediately. A RescueTime study found that we take 26 percent of our work to home [24]. Individuals may decide that health can wait if it does not hold the same urgency. Computing systems that can help the user decide what actions are the best choices given their health state may aid in these situations [17]. Unfortunately, these systems alone cannot decide how to proceed with life balance since the user ultimately must prioritize what they want.

This paper examines the conflicting dynamic between work stressors and the biological impact in the short term and long term, or what we call work-health balance. We have developed a method for conceptually characterizing one's current health state and health potential concerning both their 
work stress and healthy lifestyle design. Individuals within this system will be referred to as users of the system.

\section{WORK IMPACT ON USER Biological State}

Work causes an impact to both one's psychological or perceptual stress levels and also a change in their lifestyle events, ultimately leading to an impact to the biological state [16]. We review both the psychological components as well as lifestyle components.

\section{A. Psychological Demands}

Psychological stress includes the unpredictable, uncontrollable, and overloaded nature that users find within their lives. The Perceived Stress Scale (PSS) is the most widely used psychological instrument for measuring stress perception. It is a measure of the degree to which situations in one's life are appraised as stressful [6]. As urgent work tasks may arise or users feel overwhelmed by tasks at hand, this induces a stress response, inducing a perceived burden of executing and meeting expectations. The perceived stress to toggle between work and health remain outwardly understood by the user; however, the biological effects may or may not be immediately noticeable. The perceived stress and imbalance are harming the biological state, and this continues to feed into further impact on one's work and, therefore, further stress. This feedback loop is described in Figure 1.

User response to work-health balance disruption may be rooted in underlying psychological demands. We explore highimpact psychological factors that become behavioral patterns over time. Many of these patterns can be attributed to the individual's specific motivations [29], [18].

1) Self-Expectations: Users may have set goals or selfexpectations for their performance and their execution on their work. These expectations may arise in various forms and have a powerful influence in making decisions on work and health. If urgent tasks arise, self-expectations may cause users to favor the work domain over their health. In this situation, health continues to be neglected if the user does not set the same expectation around maintaining or improving their lifestyle.

2) External Influences: Social expectations play a role in contributing to the user's decision-making process (i.e., leaving work to exercise). A component of user decisionmaking for maintaining balance with work arises from external influences, such as managers, colleagues, family, or peers. Psychologically, users may place value in others' perceptions of their work dedication. Fear of disappointment becomes a factor in considering how to manage one's time appropriately [9].

The failure to communicate life priorities and personal boundaries makes creating a healthy balance with work more challenging. Often, users face fears of expressing the need for flexibility in work hours or the need for timeline extensions and the inability to meet an expected deadline.

While internal factors play a role in life satisfaction, environmental factors play a role in psychological demands and barriers that impact stress. A study conducted on satisfaction as a teacher was related to internal factors, such as satisfaction in life in general and feelings of self-efficacy, while reported stress was related to external factors, such as principal and pupil ratings [28]. Users may be highly influenced by their environment, which is why physical and social surroundings influence many lifestyle diseases. For example, office happy hours may influence individuals to drink more than they typically would. Individuals may perceive that they are operating with "free choice," however, the environment influences the choices they make and may lead them to act in ways counter to true preferences, a detriment to taking action toward a healthy lifestyle [27].

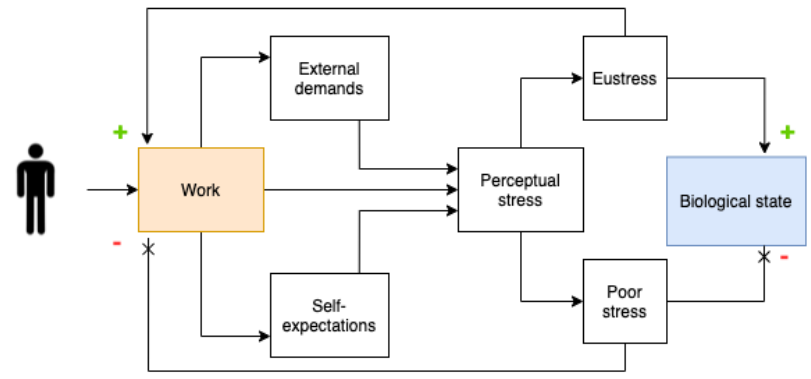

Fig. 1. Psychological Demands Impacting Health State: There are psychological demands, including external demands and self-expectations, that ultimately lead to perceived stress. This stress may be a eustress, or ideal stress that can have positive effects to one's biological state and functioning [13], [15]. If the stress is negative or "poor stress," it harms the biological state, which in turn harms the user's ability to work most effectively.

3) Temporal Behaviors: When urgency arises from demanding work needs, users may engage in temporal behaviors or time-dependent behavior in which they are making decisions to seek immediate rewards. For example, meeting a work deadline is a tangible and time-dependent requirement with an immediate reward for completion. When deciding to work late, health may be sacrificed as it is a longer-term consideration as there may not be immediate changes visible.

Users most commonly operate in seeking immediate rewards for money and health rather than long term gains. This economic phenomenon is known as temporal discounting [5]. Users can plan for the longer term; however, short term benefits or changes are always more tangible. If someone knows they may have a heart attack in twenty years if they continue their current lifestyle trajectory, they may not change their current exercise regimen. Twenty years does not seem tangible and foreseeable.

As the constant battle between work and health becomes a pattern, users may find themselves seeking a vacation to "de-stress" and seek relief or a new form of reward for their hard work. Vacations and time off are becoming avenues for stress mitigation and is a temporary near-term solution, rather than a more sustainable and permanent approach to address the underlying cause of work-life imbalance. Health, in this process, continues to be negatively impacted. 


\section{B. Short-Term Biological Impact}

The effects of perceived work stress have an impact on short-term physical health. Job stress has a causal impact on ill physical health [7]. These effects include acute biological stress and an increase in cortisol levels [23] and harmful impact on physiologic exercise recovery and sleep disturbance, impacting psychological and physiological function demonstrated in a study conducted in soccer players [20]. Individuals with higher levels of job stress notice a detrimental change in diet patterns [31]. Lastly, we see an impact on mental health from direct increases in anxiety and mood fluctuation from working excessively long hours [22]. Research shows that employees who monitor their work email frequently outside of work report higher levels of anxiety [3]. These mentioned short-term biological changes are generally felt by the user daily when not in balance.

\section{Long-term biological impact}

Continuous stressful work in one's lifestyle has longer-term effects, including an increased risk of metabolic disease, which may not be apparent to users immediately. While health is negatively impacted over time, users may be diminishing their health potential, or total ability to function. Research in health psychology and behavioral medicine indicates that physiological stress reactions bring about the risk of disease [10]. Stressors, especially with a threat that is unremitting in older and unhealthy individuals, can damage health in the long term. "The relationship between psychosocial stressors and disease is affected by the nature, number, and persistence of the stressors as well as by the individual's biological vulnerability (i.e., genetics, constitutional factors), psychosocial resources, and learned patterns of coping" [26]. The persistence in this case of work stressors can contribute to the course of longer-term chronic diseases, including cardiovascular disease [10] and the impact of ongoing diet disturbance that lead to hypercholesterolemia [31].

\section{Characterizing Scenarios that Cause WORK-HEALTH IMBALANCE}

We split the user's health state as a result of work impact through the lens of psychological stress and daily lifestyle. This is shown in stress versus lifestyle matrix in Figure 2, which can help us visually observe where a user depends on both their lifestyle components and their stress levels with work. This personalized situation can be modeled using event mining, as shown in the literature on lifestyle parameters such as sleep quality [30]. Specific examples of users in relation to the quadrant location on the matrix are shown in I.

We can next project a user's health trends over a shortterm based on lifestyle and stress. Figure 3 shows the short term impact on the biological state. We review impacts to health by where the user is located on the health continuum, a spectrum of high quality of life to developing dysfunction [17], [29]. Graph (a) corresponds to users with poor lifestyle and poor stress, contributing to a decrease in the health state over months, or a negative balance. Graph (b) corresponds

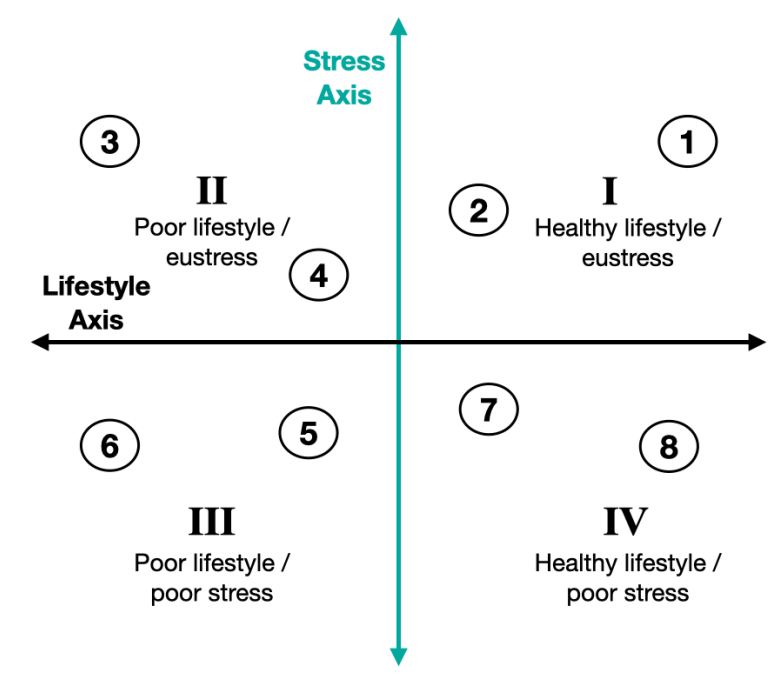

Fig. 2. Lifestyle-Stress Matrix: In this matrix, Quadrant I represents healthy lifestyle and eustress, Quadrant II represents poor lifestyle and eustress, Quadrant III represents poor lifestyle and poor stress, and Quadrant IV represents healthy lifestyle and poor stress.

\begin{tabular}{ll}
\hline$\#$ & Example \\
\hline 1 & Joyful mother who hikes every morning \\
2 & Professional athlete \\
3 & Living by the beach with family without exercise \\
4 & Traveling the world without consistent sleep \\
5 & Staying late at work with no time for exercise \\
6 & Stressed about unemployment and eating junk food \\
7 & Training for marathon and staying at work late \\
8 & Maintaining healthy diet but stressed about work deadline \\
\hline
\end{tabular}

TABLE I

THIS TABLE SHOWS EXAMPLES OF REAL-LIFE SITUATIONS PLACED ON THE LIFESTYLE-STRESS MATRIX. THE EXAMPLE NUMBER CORRESPONDS TO THE LOCATION WITHIN THE QUADRANTS IN FIGURE 2.

to negligible impacts on health within the short-term, and therefore a static trend. This indicates that the user is on the borderline of entering a poor health state. Lastly, graph (c) corresponds to a positive balance in the health state, in which there are periods of highly stressful times at work or temporary costs to health, but overall a positive trend towards a greater health state. While fluctuations may occur, it is important to observe the moving average of the health state over time. In the best case, users will continue to increase towards their maximum health potential.

Next, we want to observe what happens to the user's total health potential given patterns of their work stress and health over the long-term, including the span of their lifetime. The health potential encompasses the potential capabilities of one's health state [16]. For example, if we observe a user's cardiovascular state, all possible measurements for the cardiovascular state will be considered, including functional output and disease risk components. Once users reach an irreversible 


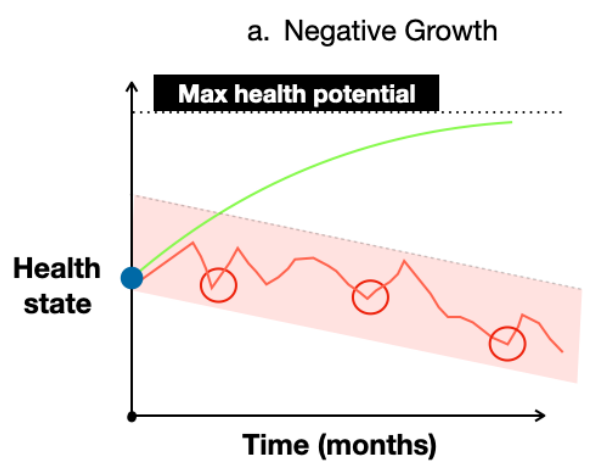

Developing Dysfunction b. Static

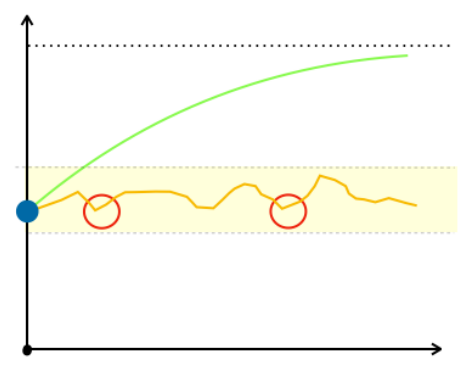

Time (months) c. Positive Growth

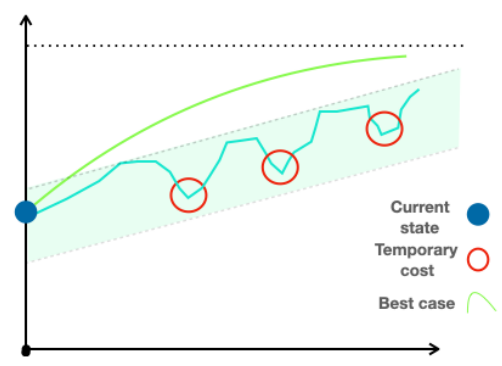

Time (months)
Higher quality of life

Fig. 3. Health State Outcomes in the Short-Term: We see various possibilities for how the health state is impacted by lifestyle and stress components and where these situations are along the health continuum. a) A negative balance in health state due to poor lifestyle and poor stress. b) Static balance in health state due to negligible impacts to health from lifestyle and stress factors. c) A positive balance in health state due to positive lifestyle and stress factors.

threshold, they may no longer be able to return to their former health state, as shown in Figure 4. An example would be a user who is irreversibly diagnosed with cardiovascular disease or metabolic syndrome. This person will now need to rely on medications for their remaining lifetime. Once a threshold of disease is reached, there is no longer a point of return to the prior health potential.

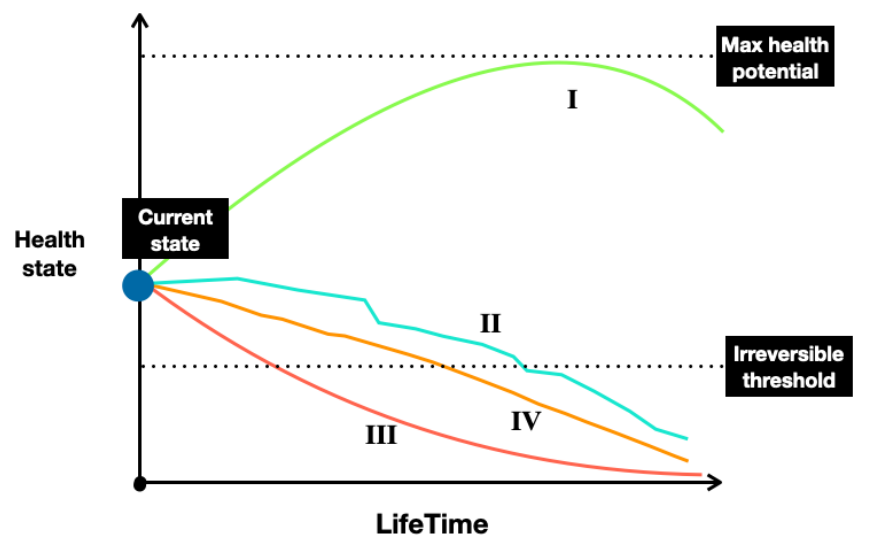

Fig. 4. Health State Outcomes over Lifetime: Over one's lifetime, we can observe that if poor work stress or poor lifestyle are regularly part of a user's life, their health will deteriorate more quickly and approach an irreversible threshold for their health potential. Based on users within the Lifestyle-Stress Matrix described in 2, we see that Quadrant III, or users with poor lifestyle and poor stress, deteriorate in health most rapidly. Users in Quadrant I, or good health and a good lifestyle, maintain health closer to their maximum health potential over a longer period. Quadrant II and IV may have unpredictable outcomes.

\section{A. N-of-1 Experiment: Personal Monitoring Work Impact to Health}

We collected data on a single user, an N-of-1 modeling approach, over 51 weeks to observe lifestyle health through exercise and objective stress. Figure 5 shows the moving average of the data over time. Lifestyle exercise data (i.e., walking, running, cycling) is computed through TriScore Loading [11] and stress data derived from heart rate variability from Garmin Fenix 5 to track changes over time. We then took these measurements and overlay stressful work deadlines within the year to see if lifestyle and stress have impacts correlated with the work deadlines.

Our findings show that during periods of stressful deadlines, both lifestyle events and stress are negatively impacted. From weeks 16 to 24 , we see a drop in lifestyle activities and an increase in stress, leading to overall negative stress and lifestyle trends, as shown in the graph's red region. Similarly, weeks 32 to 44, leading up to a work deadline in Week 44, also shows a negative impact on both lifestyle and stress. In both situations, once the work deadline passes, both lifestyle and stress begin to improve steadily. It is important to note that there may be additional stressful confounding factors outside of work that may impact the stress levels.

\section{CONCLUSION}

Work-health balance is critical for a user's longer-term wellbeing. The method presented can characterize work impact on health, and could be highly valuable to employee/employer decisions on workplace policies considering employee work stress and its impact on their health over time. Our method for characterizing health state relationships to work can also allow users to see highly personalized and data-driven approaches for their work and health decisions as they build towards designing their lifestyles. The method described in this paper is an initial framework to define work-health balance that can be expanded upon with further complexities.

\section{REFERENCES}

[1] The Council of Economic Advisers. WORK-LIFE BALANCE AND THE ECONOMICS OF WORKPLACE FLEXIBILITY. White House (President Barack Obama), 2014.

[2] Amanda S. Bell, Diana Rajendran, and Stephen Theiler. Job stress, wellbeing, work-life balance and work-life conflict among Australian academics. Technical Report 1, 2012. 


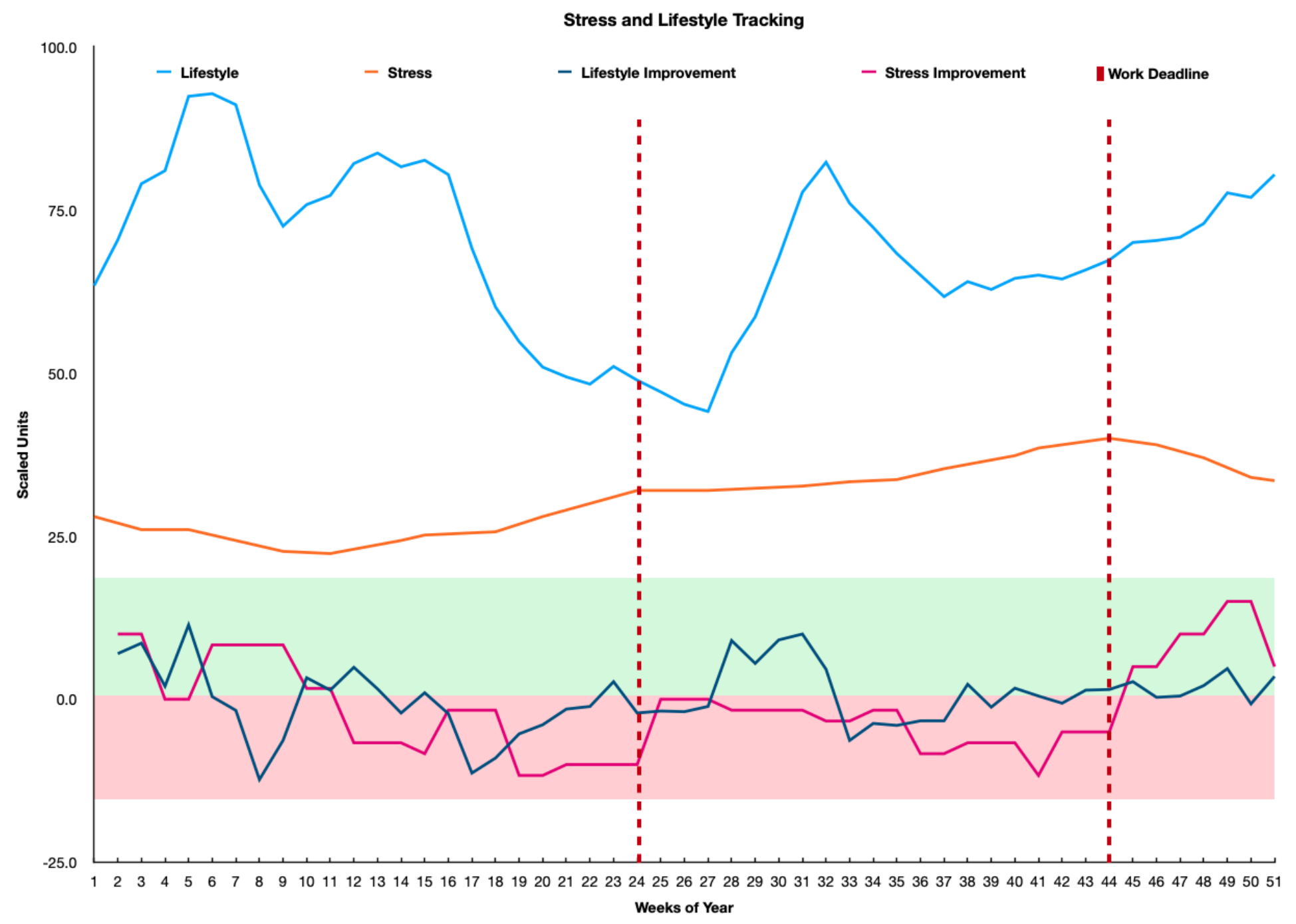

Fig. 5. Stress and Lifestyle Tracking: We collected over 51 weeks of user data tracking lifestyle events, stress, and the change week-over-week in both lifestyle and stress. We also show the major work deadlines in the year (Week 24 and 44). The green region shows the improvement week over week and the red region shows a decrease week by week. The trends give us information about how both stress and lifestyle change and when this is happening relative to the significant work events.

[3] Lehigh Business. Answering Emails After Work Can Stress Relationships and Harm Health.

[4] Sarah Green Carmichael. The Research Is Clear: Long Hours Backfire for People and for Companies. Harvard Business Review, 2015.

[5] Gretchen B. Chapman. Temporal discounting and utility for health and money. Journal of Experimental Psychology: Learning Memory and Cognition, 22(3):771-791, 1996.

[6] Sheldon Cohen, T Kamarck, and R Mermelstein. Perceived Stress Scale. Technical report, 1983.

[7] Cary L. Cooper, Bruce D. Kirkcaldy, and Jennifer Brown. A model of job stress and physical health: the role of individual differences. Personality and Individual Differences, 16(4):653-655, 41994.

[8] Margaret Deery and Leo Jago. Revisiting talent management, work-life balance and retention strategies. International Journal of Contemporary Hospitality Management, 27(3):453-472, 42015.

[9] Gary Alan Fine and Leonard Berkowitz. Advances in Experimental Social Psychology, Vol. 17., volume 14. Elsevier, 1985.

[10] Peter J. Gianaros and Tor D. Wager. Brain-Body Pathways Linking Psychological Stress and Physical Health. Current Directions in Psychological Science, 24(4):313-321, 82015.

[11] GoldenCheetah. GoldenCheetah Version 3.5, 2020.
[12] Jarrod M. Haar, Marcello Russo, Albert Suñe, and Ariane OllierMalaterre. Outcomes of work-life balance on job satisfaction, life satisfaction and mental health: A study across seven cultures. Journal of Vocational Behavior, 85(3):361-373, 122014.

[13] Mark Le Fevre, Jonathan Matheny, and Gregory S. Kolt. Eustress, distress, and interpretation in occupational stress, 2003.

[14] Thorsten Lunau, Clare Bambra, Terje A Eikemo, Kjetil A van der Wel, and Nico Dragano. A balancing act? Work-life balance, health and wellbeing in European welfare states. European Journal of Public Health, 2014.

[15] John H. Milsum. A model of the eustress system for health/illness Behavioral Science, 30(4):179-186, 11985.

[16] Nitish Nag. Health State Estimation. $\mathrm{PhD}$ thesis, University of California, Irvine, 32020.

[17] Nitish Nag and Ramesh Jain. A Navigational Approach to Health: Actionable Guidance for Improved Quality of Life. Computer, 52(4):1220, 122019.

[18] Nitish Nag, Mathias Lux, and Ramesh C. Jain. Intrinsic and Extrinsic Motivation Modeling Essential for Multi-Modal Health Recommender Systems. Arxiv, 82018.

[19] Nitish Nag, Vaibhav Pandey, Preston J. Putzel, Hari Bhimaraju, Srikanth 
Krishnan, and Ramesh Jain. Cross-Modal Health State Estimation. In MM 2018 - Proceedings of the 2018 ACM Multimedia Conference, pages 1993-2002, New York, New York, USA, 10 2018. Association for Computing Machinery, Inc.

[20] Mathieu Nédélec, Shona Halson, Abd Elbasset Abaidia, Said Ahmaidi, and Gregory Dupont. Stress, Sleep and Recovery in Elite Soccer: A Critical Review of the Literature, 102015.

[21] Leslie A. Perlow and Jessica L. Porter. Making Time Off Predictable-and Required. Harvard Business Review, 2009.

[22] Barbara Pocock. The Work/Life Collision. The Federation Press, 2003.

[23] Jens C. Pruessner, Dirk H. Hellhammer, and Clemens Kirschbaum. Burnout, perceived stress, and cortisol responses to awakening. Psychosomatic Medicine, 61(2):197-204, 1999.

[24] RescueTime. The State of Work Life Balance in 2019.

[25] Doris Ruth Eikhof. Introduction: What work? What life? What balance? Employee Relations, 29(4), 72007.

[26] Neil Schneiderman, Gail Ironson, and Scott D. Siegel. Stress and Health: Psychological, Behavioral, and Biological Determinants. Annual Review of Clinical Psychology, 1(1):607-628, 42005.

[27] Harvard Medical School. From the journals: Four small lifestyle changes can mean an extra 14 years, 2008.

[28] J. SMILANSKY. EXTERNAL AND INTERNAL CORRELATES OF TEACHERS' SATISFACTION AND WILLINGNESS TO REPORT STRESS. British Journal of Educational Psychology, 54(1):84-92, 2 1984.

[29] Tabya Sultan and Nitish Nag. Motivation and Joy Mapping for Sustainable Healthy Lifestyle Design. MindRxiv, page 8, 2020.

[30] Dhruv Upadhyay, Vaibhav Pandey, Nitish Nag, and Ramesh Jain. N=1 Modelling of Lifestyle Impact on SleepPerformance. Arxiv, 62020.

[31] E. Leigh Wardle, Jane Gibson. Impact of stress on diet: Processes and implications, 2019. 